\title{
Autophagy-active beclin-1 correlates with favourable clinical outcome in non-Hodgkin lymphomas
}

Giuseppina Nicotra ${ }^{1,2}$, Francesca Mercalli ${ }^{2}$, Claudia Peracchio ${ }^{1}$, Roberta Castino ${ }^{1}$, Carlo Follo ${ }^{1}$, Guido Valente ${ }^{2}$ and Ciro Isidoro ${ }^{1}$

${ }^{1}$ Laboratorio di Patologia Molecolare del Dipartimento di Scienze Mediche, Novara, Italy and ${ }^{2}$ Laboratorio di Anatomia Patologica del Dipartimento di Medicina Clinica e Sperimentale, Università del Piemonte Orientale 'A Avogadro', Novara, Italy

The expression of beclin-1, an oncosuppressor monoallelically deleted in $>60 \%$ epithelial cancers, has been shown to be developmentally regulated in T and B lymphocytes. By interacting with either bcl-2 or class III phosphatidyl-inositol-3-phosphate kinase, beclin-1 regulates apoptosis and autophagy, two processes crucial for lymphatic tissue homeostasis. We analyzed the potential link between beclin-1-mediated autophagy and the malignant behaviour of lymphomas. The tissue expression of beclin-1 was analyzed in a large series of nonHodgkin lymphomas and correlated with patient's clinical outcome. By immunofluorescence, beclin-1 staining showed faintly detectable and diffusely distributed in the cytoplasm (regarded as negative) or confined to the perinuclear region as large and brilliant puncta suggestive of macro-aggregate reactivity (regarded as positive). The positive expression of beclin-1 well correlated with the presence of LC3-positive autophagic vacuoles and was inversely correlated with the expression of bcl-2. Non-Hodgkin lymphomas in which $\geqslant 20 \%$ of tumour cells expressed high level of beclin-1 aggregates were associated with a complete $(57 \%)$ or partial $(35 \%)$ remission. The 5-year overall survival probability, calculated by the Kaplan-Meier method, was $92 \%$ and $42 \%$ in beclin-1expressing non-Hodgkin lymphomas with $\geqslant 20 \%$ and $<20 \%$ positive cells, respectively (log-rank test, $P<0.000 .1)$. In Cox multivariate analysis, the level of beclin-1 expression, adjusted for patient's age and pathologic stage, revealed to be significantly correlated with patient's survival $(P<0.0001)$. This is the first demonstration of the involvement of beclin-1 and autophagy in the clinical behaviour of non-Hodgkin lymphomas. The present data are compatible with the hypothesis that non-Hodgkin lymphomas with upregulated autophagy are more responsive to chemotherapy and indicate that beclin-1 could be a valuable independent prognostic factor in this heterogeneous group of tumours.

Modern Pathology (2010) 23, 937-950; doi:10.1038/modpathol.2010.80; published online 14 May 2010

Keywords: autophagy; beclin-1; LC3; bcl-2; tumour marker; prognosis

The beclin-1 gene is reportedly monoallelically deleted in a large proportion of prostate, breast and ovary cancers, ${ }^{1-5}$ whereas its homozygous deletion has never been found in either normal or tumour tissues. The beclin-1 gene is regarded as a haploinsufficient tumour suppressor, because its heterozygous deletion in transgenic mice favours the

Correspondence: Professor C Isidoro, $\mathrm{MD}, \mathrm{PhD}$, Dipartimento di Scienze Mediche, Università 'A Avogadro', Via Solaroli 17, 28100 Novara, Italy.

E-mail: isidoro@med.unipmn.it

Received 13 November 2009; revised 16 March 2010; accepted 24 March 2010; published online 14 May 2010 spontaneous development of tumours in different tissues. ${ }^{6,7}$ The gene codes for a $60 \mathrm{kDa}$ protein that was first identified in a yeast two-hybrid system screen as an interactor of the anti-apoptotic protein bcl-2. ${ }^{8}$ Through its interaction with class III phosphatidyl-inositol-3-phosphate kinase (PI3kIII/ Vps34), beclin-1 (also known as atg6/Vps30) regulates autophagy. ${ }^{9,10}$ Autophagy is a lysosomaldependent pathway for macromolecular and organelle degradation that has a pivotal role in cell homeostasis. ${ }^{11}$ Autophagy begins with the entrapment of the substrate (protein aggregates, aged and oxidized membranes or organelles) by a multilayered membrane originated in the proximity 
of the endoplasmic reticulum and trans Golgi network. ${ }^{12}$ The resulting vesicle, named the 'autophagosome', is marked by the insertion into its membranes of the protein LC3-II (also known as atg8) that arises, by partial proteolysis and subsequent lipidation, from the microtubule-associated protein light chain isoform I (LC3-I).$^{13}$ Subsequently, by fusing with endosomal/lysosomal compartments, the autophagosome matures into the autophagolysosome, wherein the substrate is degraded by acid hydrolases. ${ }^{14}$ Autophagy is triggered by the so-called beclin-1/PI3kIII interactome, a macro-complex participated by several other proteins, which locates in the proximity of the trans Golgi network. ${ }^{12}$ The autophagy process is finely tuned by sensors of nutrients and energy available. ${ }^{15}$ Stressful and potentially toxic environmental stimuli, such as nutrient and growth factors depletion or pro-oxidant radicals, induce autophagy. By removing oxidatively damaged proteins and organelles and by providing substrates and energy through the catabolism of redundant cellular components, autophagy confers a pro-survival advantage to the cell exposed to potentially adverse intra- and extracellular situations. ${ }^{16}$ However, the interplay between autophagy and the cell survival and cell death pathways is quite complex. In fact, depending on the nature of the stimulus and the actual metabolic status of the cell, autophagy can suppress or enable apoptosis. ${ }^{17}$ Clearly, the crosstalk between the autophagy and apoptosis signalling pathways is critical to the overall fate of the cell, and beclin-1, owing to its ability to interact with either PI3kIII or bcl-2, occupies a key position in this crosstalk. ${ }^{18}$ Not surprising, autophagy has been shown to have an active role in cancer development and progression, ${ }^{19,20}$ as well as in the cytotoxic response to anticancer drugs. ${ }^{21-24}$

In $\mathrm{T}$ lymphocytes autophagy has been implicated in survival and cytokine-induced proliferation, as well as in cell death after growth factor-withdrawal. ${ }^{25,26}$ The expression level of beclin-1 changes during $\mathrm{T}$ and $\mathrm{B}$ lymphocyte maturation and T-cell activation. ${ }^{27}$ Fluctuation of beclin-1 expression was shown to parallel that of bcl-2 during the maturation process of $\mathrm{T}$ cells in the thymus and of $\mathrm{B}$ cells in bone marrow. ${ }^{27}$ In addition, overexpression of ectopic beclin-1 in thymocytes exacerbates glucocorticoid-induced apoptosis. ${ }^{27}$ Altogether these data imply that autophagy has a critical role in lymphocyte homeostasis. Consistent with such a role, transgenic mice lacking one beclin-1 allele present with a reduced autophagy activity in the lymph node germinal centre and show a high incidence of spontaneous lymphomas of the B lineage. ${ }^{6,7}$

In this work, we focused on the relationship between beclin-1 expression and the clinical behaviour of nonHodgkin lymphomas. Our data show the involvement of autophagy in the post-therapy outcome in nonHodgkin lymphoma-bearing patients and support the usage of beclin-1 as a reliable prognostic factor in this heterogeneous group of tumours.

\section{Materials and methods}

\section{Patients and Tissue Biopsies}

The study included 102 cases of non-Hodgkin lymphomas selected in the years 2000-2008 from the files of the Department of Pathology of Università del Piemonte Orientale (Novara, Italy). Cases were randomly selected, not knowing in advance the clinical outcome, or the therapy regimen, and not considering specific clinical presentation (such as the performance status, immunodeficiency, pathologic stage, and so on) of the patient at diagnosis. Cases included in the study were of de novo diagnosis, that is, not derived from relapse or malignant progression of previously diagnosed and cured lymphomas. All cases were classified according to the current WHO Classification of Lymphoid Neoplasms. ${ }^{28}$ The following cases, chosen among the non-Hodgkin lymphomas diagnosed in our department in the considered period, were available for this study (WHO abbreviation; site of biopsy): no. 17 small lymphocytic lymphomas (SLLs; lymph node), no. 32 diffuse large B-cell lymphomas (DLBCLs; lymph node), no. 9 follicular lymphomas of grade I and II (FLs I and II; lymph node) and no. 12 FLs of grade IIIB (FLs IIIB; lymph node), no. 11 marginal zone lymphomas (MZLs; three lymph node, eight spleen), no. 16 mucosa-associated lymphatic tissue lymphoma (MALToma; four stomach, three skin, three parotid, three orbit, one breast, one oropharynx, one rinopharynx) and no. 5 peripheral T-cell lymphomas (PTCLs, lymph node). Biopsies were obtained from patients at diagnosis, that is, before any therapeutic intervention.

\section{Tissue Immunofluorescence}

Formalin-fixed paraffin-embedded tissue sections were cut from all non-Hodgkin lymphoma cases. After extensive removal of paraffin, the sections were incubated first with the primary antibody (diluted in phosphate-buffered saline supplemented with $0.1 \%$ Triton X-100 and $4 \%$ fetal calf serum, for $16 \mathrm{~h}$ in a humid chamber at $4{ }^{\circ} \mathrm{C}$ ) and then with Texas Red- or FITC-conjugated secondary antibodies (diluted 1:200 as above, for $1 \mathrm{~h}$ at room temperature in a humid chamber). Excess of unbound antibody was removed at each step by two washes with phosphate-buffered saline. As negative control, incubation with the primary antibody was omitted and the section was incubated with the secondary antibody alone. The following primary antibodies were used: (1) mouse monoclonal anti-beclin-1 (BD Pharmingen, San Diego, CA, USA; dilution 1:100); (2) mouse monoclonal anti-bcl2 (Santa Cruz Biotechnology, Santa Cruz, CA, USA; dilution 1:100); and (3) rabbit polyclonal anti-LC3 (Novus Biological, Littleton, CO, USA; dilution 1:500). As secondary antibodies, goat-anti-mouse IgG or goat-anti-rabbit 
IgG (Sigma-Aldrich, St Louis, MO, USA; dilution 1:200) were used as appropriate.

The nucleus was evidenced by staining the chromatin with the fluorescent dye 4,6-diamidino2-phenylindol-dihydrochloride (DAPI, 1:500 from a stock solution $20 \mathrm{mg} / \mathrm{ml} ; 1 \mathrm{~h}$ ). Stained sections were mounted with Slow-FAD (Light AntiFADE Kit, Molecular Probes Invitrogen, Carlsbad, CA, USA) and observed under a fluorescence microscope (Leica DM1600, Leica Microsystem, Heidelberg, Germany) equipped with a digital camera. Each biopsy was tested at least three times. Representative areas were imaged using a microscope equipped with a digital camera. Cells were identified by DAPI staining of the nucleus. The proportion of positively stained (in terms of beclin-1 macro-aggregates) over total cells present in the imaged areas were expressed as percentage. At least five fields (approximately 5000 cells) per section were evaluated independently by three investigators (GV, GN and CI). Infiltrating macrophages and T-lymphocytes were identified with anti-CD68 (clone KP1, Dako, Glostrup, Denmark) and anti-CD3 (clone PS1, Novocastra, Leica Biosystem, UK) monoclonal antibodies, respectively.

\section{Tissue Western Blotting}

Pieces from frozen biopsy were cut and finely homogenized by several cycles of freeze-thawing and sonication in a phosphate buffer containing detergents (Triton X-100 and Na-deoxycholate) and a cocktail of protease inhibitors. A $50 \mu \mathrm{g}$ of protein homogenate was resolved by SDS-polyacrylamide $(13.5 \%)$ gel electrophoresis under reducing conditions. Electrotransfer and immunodetection were carried out as previously described. ${ }^{29}$ Beclin-1 and bcl-2 polypeptides were detected, respectively, with a monoclonal antibody (BD Pharmingen; dilution 1:250) and a rabbit polyclonal antiserum (Santa Cruz Biotechnology; dilution 1:100). Immunocomplexes were revealed by incubation with peroxidase-conjugated goat-anti-mouse or anti-rabbit (as appropriate) antibody (Sigma-Aldrich; dilution 1:20.000), and subsequent peroxidase-induced chemiluminescence reaction (Bio-Rad, Hercules, CA, USA). Equal loading among lanes was ascertained by re-probing the filter (after stripping and washing) with an antiactin monoclonal antibody (Sigma-Aldrich; dilution 1:250) followed by incubation with peroxidaseconjugated goat-anti-mouse antibody and revealed as above.

\section{Statistical Analysis}

Beclin-1 and LC3 expression were correlated to the clinical outcome calculating the odds ratio, the relative risk and the $\chi^{2}$. The Fisher's exact test was also used for pairwise comparison of distributions of categorized groups. The probability of overall survival for patients, as related to the expression of beclin-1, was estimated by the Kaplan-Meier method. ${ }^{30}$ Overall survival was calculated from the date of initial diagnosis until the date of last follow-up or patient's death. Surviving patients were censored at the date of last contact and mathematically removed from the curve. The significance of differences between survival curves was calculated by using the log-rank test. To determine the relative influence of beclin- 1 and of patient's age and pathologic stage on the cumulative survival probability, univariate and multivariate analyses were also performed using the Cox proportional hazard model. These analyses yielded the coefficient of regression, the $\chi^{2}$, the hazard ratios, the confidential interval and $P$-value for each variable. To indicate statistical significance, the threshold for $P$-values was taken at $5 \%$ level. Statistics was performed using the XLSTAT 2010 software.

\section{Results}

\section{Histologic Type and Main Clinical Characteristics of Non-Hodgkin Lymphomas Included in the Study}

The study was conducted in a series of 102 cases of non-Hodgkin lymphomas that included the following histologic types: SLL (17 cases), DLBCL (32 cases), FL I and II (9 cases) and FL IIIB (12 cases), MZL (11 cases), MALToma (16 cases) and PTCL (5 cases). Histologic diagnosis was corroborated by complementary immunophenotyping with a panel of antibodies. All patients were subjected to chemotherapy and/or radiotherapy, following standard criteria based on clinical stage, histologic type and patient performance status. The following information was made available at the end of the study: patient's gender and age, clinical stage at first diagnosis, objective response to chemo- and radiotherapy, and clinical outcome. Patient's median age was 67 years. Response to therapy regimen was evaluated according to the international guidelines. Clinical outcomes were classified as complete remission (disappearance of any evidence of disease for at least 24 months), partial remission ( $\geqslant 50 \%$ decrease in tumour lesions for at least 24 months) and exitus. The histologic type and the main clinical characteristics of the cases included in this study are reported in Table 1.

\section{Immunodetection of Beclin-1 in Non-Hodgkin Lymphoma Tissue Sections}

Beclin-1 is a protein ubiquitously expressed in eukaryotic cells. When activated, beclin-1 is recruited together with other proteins to form macro-complexes that locate in the proximity of the endoplasmic reticulum and of the trans-Golgi network. ${ }^{12}$ The presence and the cytoplasmic distribution of beclin-1 were analyzed by immunofluorescence in 
Table 1 Clinico-pathological data of non-Hodgkin lymphomas included in the study

\begin{tabular}{|c|c|c|c|c|c|c|c|c|}
\hline \multirow[t]{2}{*}{ Histologic type } & \multirow[t]{2}{*}{ Sex } & \multirow[t]{2}{*}{ No. of cases } & \multirow[t]{2}{*}{ Age (range) } & \multirow[t]{2}{*}{ Median age } & \multicolumn{2}{|c|}{ Stage } & \multirow[t]{2}{*}{ CR rate } & \multirow[t]{2}{*}{ Survival rate (5 year) } \\
\hline & & & & & $I$ and II & III and IV & & \\
\hline \multirow[t]{2}{*}{ DLBCL (32) } & M & 16 & $46-79$ & 66 & $13^{*}$ & 18 & $41 \%$ & $47 \%$ \\
\hline & $\mathrm{F}$ & 16 & 27-81 & 71 & & & & \\
\hline \multirow[t]{2}{*}{ SLL (17) } & $\mathrm{M}$ & 9 & $34-81$ & 51 & 1 & 16 & $12 \%$ & $47 \%$ \\
\hline & $\mathrm{F}$ & 8 & $68-90$ & 75.5 & & & & \\
\hline \multirow[t]{2}{*}{ MALToma (16) } & M & 5 & $44-79$ & 60 & 11 & 5 & $75 \%$ & $75 \%$ \\
\hline & $\mathrm{F}$ & 11 & $49-75$ & 66 & & & & \\
\hline \multirow[t]{2}{*}{ FL IIIB (12) } & M & 5 & $35-77$ & 63 & 5 & 7 & $50 \%$ & $58 \%$ \\
\hline & $\mathrm{F}$ & 7 & $49-76$ & 68 & & & & \\
\hline \multirow[t]{2}{*}{ MZL (11) } & M & 4 & $53-74$ & 70.5 & 4 & 7 & $36 \%$ & $55 \%$ \\
\hline & $\mathrm{F}$ & 7 & $55-82$ & 68 & & & & \\
\hline \multirow[t]{2}{*}{ FL I and II (9) } & M & 1 & 69 & 69 & 2 & 7 & $22 \%$ & $11 \%$ \\
\hline & $\mathrm{F}$ & 8 & $48-73$ & 62 & & & & \\
\hline \multirow[t]{2}{*}{ PTCL (5) } & $\mathrm{M}$ & 3 & $49-73$ & 68 & 0 & 5 & $0 \%$ & $0 \%$ \\
\hline & $\mathrm{F}$ & 2 & $73-74$ & 73.5 & & & & \\
\hline
\end{tabular}

Abbreviations: CR, complete remission; DLBCL, diffuse large B-cell lymphoma; FL, follicular lymphoma (grade I, II or IIIB); MALTomas, mucosaassociated lymphatic tissue lymphoma; MZL, marginal zone lymphoma; PTCL, peripheral T-cell lymphoma; SLL, small lymphocytic lymphoma. *One lacking data.

paraffin-embedded tissue sections ( $4 \mu \mathrm{m}$ thick) of the most representative tumour area. Biopsies were obtained from patients de novo diagnosed for nonHodgkin lymphoma and not yet subjected to therapy. Non-neoplastic cells were excluded from the evaluation. In the case of doubtful interpretation between macrophages and large $B$ neoplastic cells, the sections were double-stained with the anti-beclin-1 and an anti-CD68 monoclonal antibody. In B-cell low-grade lymphomas, the presence of reactive, non neoplastic $\mathrm{T}$ lymphocytes infiltrating the lymphoma was assessed by CD3 immunostaining. Double staining with anti-beclin-1 and anti-CD68 or anti-CD3 allowed to estimate the contribution of non-neoplastic infiltrating beclin-1-positive cells and to determine the proportion of neoplastic cells expressing beclin-1 macro-aggregates within the biopsy.

Typical staining of beclin-1-negative and beclin-1positive non-Hodgkin lymphomas is shown in Figure 1. Beclin-1 immunoreactivity in tumour cells presented as faintly detectable fluorescence diffused in the cytoplasm or as discrete fluorescent spots located near the nucleus. These immunoreactivity patterns were referred to as negative or positive, respectively, in terms of beclin-1 macro-aggregates.

\section{Correlation of Beclin-1 Expression with Non-Hodgkin Lymphoma Histologic Type}

We performed an extensive analysis of beclin-1 expression and immuno-localization in 102 nonHodgkin lymphoma biopsies. On the basis of the proportion of beclin-1-positive cells within the tumour tissue, the samples were initially stratified into four ranges of positivity: $<10 \% ; 10-20 \%$; $20-40 \%$; and $>40 \%$. Only a few tumours classified in the $10-20 \%$ range of positivity and the final threshold was set at $\geqslant 20 \%$ to indicate tumours with a high proportion of beclin-1-positive cells. In all, 59 out of 102 non-Hodgkin lymphomas showed a high proportion of beclin-1-positive cells. Beclin-1-positivity was not significantly associated with a particular histologic type, although we note that a large majority ( 16 out of 21 ) of FLs showed $\geqslant 20 \%$ positive cells (Table 2). More in details, 11 out of 12 of FLs IIIB were highly expressing beclin-1 aggregates. In addition, 13 out of 16 MALTomas were highly expressing beclin-1. On the basis of clinical behaviour or on pathologic ground, the histologic types SLL, MZL, MALToma and FL I and II are classified as 'indolent' or 'low-malignant' lymphomas and the histologic types DLBCL, FL IIIB and PTCL are classified as 'aggressive' or 'highly malignant' lymphomas. ${ }^{31,32}$ The high proportion of beclin-1-positive tumour cells was not correlated with either of these histologic subgroups (60 and $55 \%$ in 'indolent' and 'aggressive' lymphomas, respectively).

\section{Beclin-1 Expression does not Correlate with Pathological Staging at Diagnosis}

We then verified whether the proportion of beclin-1positive cells was correlated with the pathologic stage at diagnosis. Of the 43 non-Hodgkin lymphomas with $<20 \%$ of beclin-1-positive cells, 12 were classified as I and II stage and 30 as III and IV stage (one lacking data); of the 59 non-Hodgkin lymphomas with $\geqslant 20 \%$ beclin-1-positive cells, 24 were of I and II stage and 35 of III and IV stage. No significant correlation was found between the positivity for beclin-1 and the pathologic stage $(P=0.3)$. 

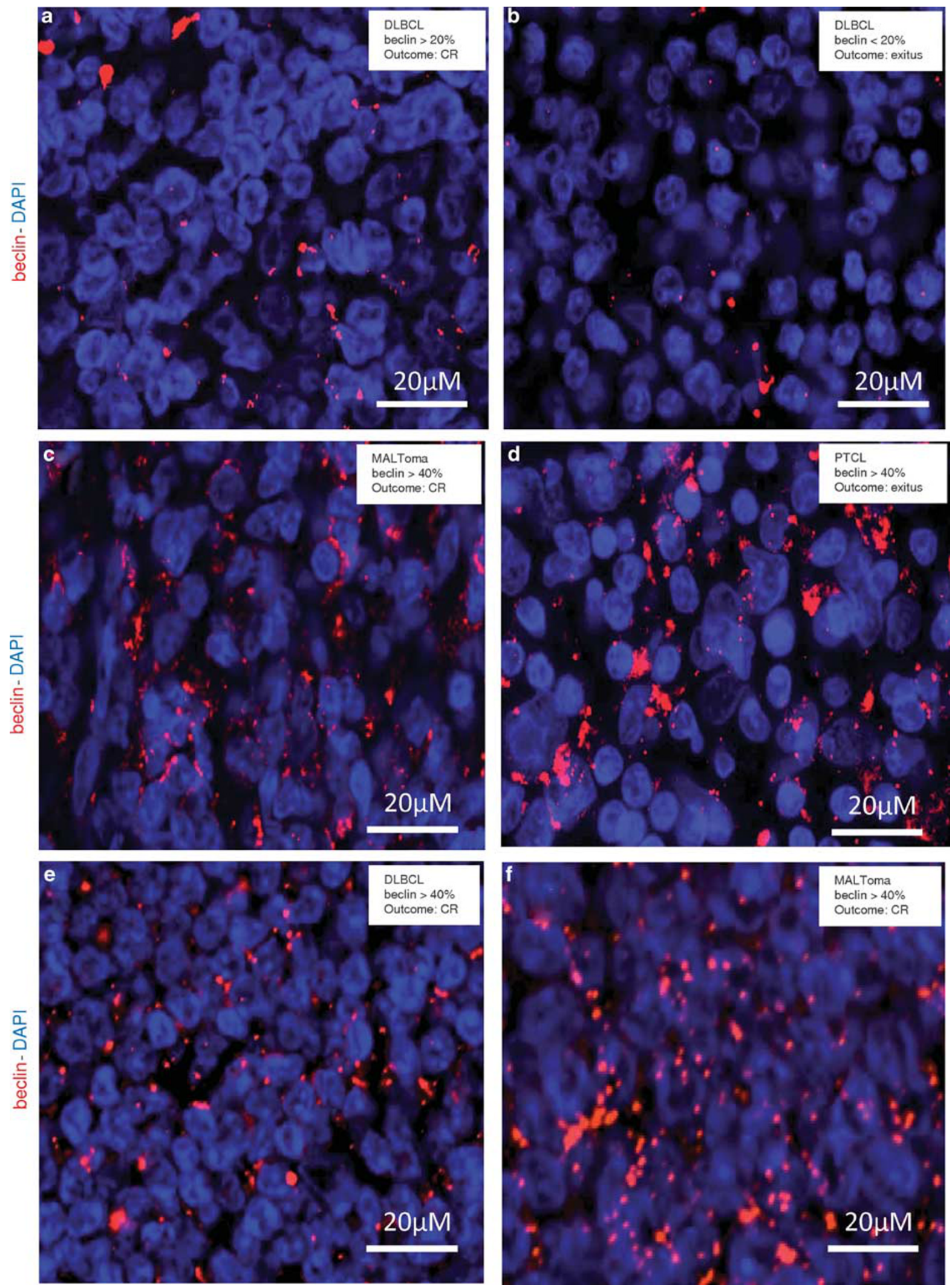

Figure 1 Immunofluorescence staining of beclin-1 in non-Hodgkin lymphoma tissue sections. Selection of representative cases. The histologic type (DLBCL, diffuse large B-cell lymphoma; MALToma, mucosa-associated lymphatic tissue lymphoma; PTCL, peripheral T-cell lymphoma), the clinical outcome (CR, complete remission; exitus) and the percentage of cells positive for beclin-1 aggregates are reported. The nuclei are evidenced by DAPI staining. Examples of beclin-1 positive (panels a, $\mathbf{c}, \mathbf{d}, \mathbf{e}$ and $\mathbf{f}$ ) and of beclin-1 negative (panel b) immunostaining. 
Table 2 Distribution of beclin-1-positivity among the histologic types of non-Hodgkin lymphoma

\begin{tabular}{|c|c|c|c|c|c|c|c|c|}
\hline Histologic type & $D L B C L$ & $S L L$ & MALToma & $F L ~ I I I B$ & $M Z L$ & FL I and II & PTCL & No. of cases \\
\hline \multicolumn{9}{|c|}{ Beclin expression } \\
\hline$<20 \%$ & 17 & 7 & 3 & 1 & 7 & 4 & 4 & 43 \\
\hline$\geqslant 20 \%$ & 15 & 10 & 13 & 11 & 4 & 5 & 1 & 59 \\
\hline Total & 32 & 17 & 16 & 12 & 11 & 9 & 5 & 102 \\
\hline
\end{tabular}

Table 3 Correlation of beclin-1-positivity in non-Hodgkin lymphomas with clinical outcome in patients

\begin{tabular}{lrrrr}
\hline Clinical outcome & CR & PR & Exitus & No. of cases \\
\hline Beclin expression & & & & \\
$\quad<20 \%$ & 6 & 13 & 24 & 43 \\
$\geqslant 20 \%$ & 33 & 21 & 5 & 59 \\
Total & 39 & 34 & 29 & 102 \\
\hline
\end{tabular}

Abbreviations: CR, complete remission; PR, partial remission.

$\chi^{2}=31.28$

$\mathrm{DF}=2$.

$P<0.0001$

\section{Beclin-1 is a Predictor of Post-Therapy Clinical Outcome in Non-Hodgkin Lymphomas}

We performed a statistical analysis to correlate the extent of beclin-1 expression (percentage of cells with beclin-1 aggregates) with the clinical outcome in the whole patient population. We note that 54 out of $73(74 \%)$ of the patients alive at the end-point of the study were bearing a non-Hodgkin lymphoma with a high proportion of beclin-1-positive cells (Table 3). Only 5 out of $59(8 \%)$ patients bearing a non-Hodgkin lymphoma with a high proportion of beclin-1-positive cells died during the observation period. Conversely, as many as 24 out of the 29 $(83 \%)$ patients who died within the period of observation were bearing a non-Hodgkin lymphoma with a low proportion of beclin-1-positive cells (Table 3). These correlations were statistically significant $(P<0.0001)$. Among the 102 non-Hodgkin lymphoma cases, 39 patients (38\%) underwent complete remission. Of these, as many as $33(85 \%)$ were bearing a non-Hodgkin lymphoma with a high proportion of beclin-1-positive cells. Conversely, only 6 out of $43(14 \%)$ patients bearing a nonHodgkin lymphoma with a low percentage of beclin1-positive cells underwent complete remission. These correlations were statistically significant $(P<0.0001)$. Of the 43 cases with a low proportion of beclin-1-positive tumour cells, as many as 24 $(56 \%)$ died and $13(30 \%)$ experienced a partial remission, whereas of the 59 cases with a high proportion of beclin-1-positive tumour cells, only 5 $(8 \%)$ died and $21(36 \%)$ experienced a partial remission (Table 3). These correlations were statistically significant $(P<0.0001)$. Thus, although a partial remission occurred with a similar frequency in patients bearing either a highly beclin-1-expressing or a low beclin-1-expressing non-Hodgkin

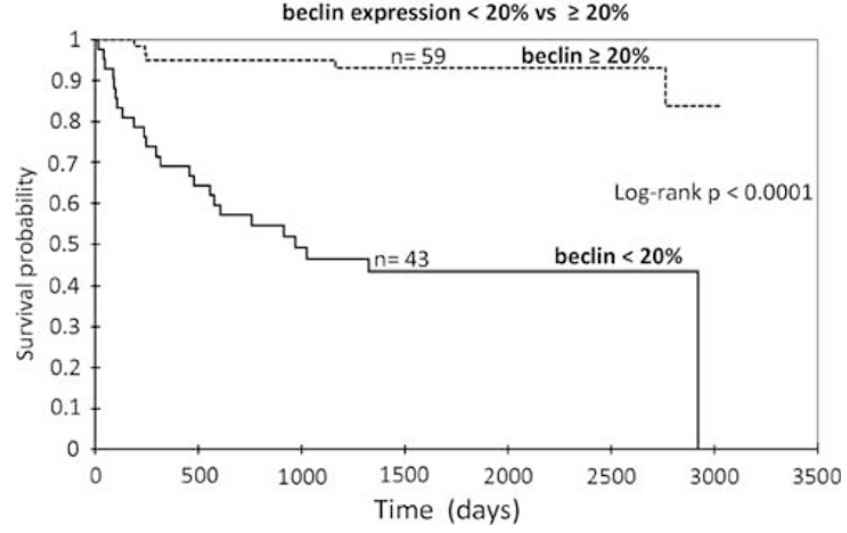

Figure 2 Kaplan-Meier curves of overall survival probability in the subgroups of patients bearing a non-Hodgkin lymphoma with less $(<)$ or more $(\geqslant)$ than $20 \%$ of cells positive for beclin-1 aggregates. The $P$-value calculated by the log-rank test is indicated.

lymphoma, complete remission occurred more frequently in the group of patients bearing a nonHodgkin lymphoma with a high percentage of beclin-1-positive cells (56 vs 14\%) and exitus was observed more frequently in the group of patients bearing a non-Hodgkin lymphoma with a low percentage of beclin-1-positive cells (56 vs 8\%).

\section{Beclin-1 is a Prognostic Marker of Malignancy in Non-Hodgkin Lymphomas}

To definitively assess the potential of the proportion of beclin-1-positive tumour cells to convey prognostic information in non-Hodgkin lymphomas, we estimated the overall survival probability in the patient population by the Kaplan-Meier method. Figure 2 shows the Kaplan-Meier curves of the cumulative probability of survival of non-Hodgkin lymphoma-bearing patients in relation to the percentage of beclin-1-positive cells within the neoplastic tissue. Log-rank test was applied to evaluate the statistical significance of such correlation.

Patients bearing a tumour that presents with a high percentage $(\geqslant 20 \%)$ of beclin-1-positive cells show a $96 \%$ overall survival probability at 2 year (93\% at 5 year and $84 \%$ at 8 year). By contrast, a low percentage of beclin-1-positive cells within the neoplastic tissue significantly associated with a low probability of overall survival of affected patients. In particular, in these patients the 2-year 
Table 4 Cox regression analysis of the influence of beclin-1 expression and patient's age and pathologic stage

\begin{tabular}{|c|c|}
\hline Prognosticator & Univariate $\mathrm{P}$ \\
\hline \multicolumn{2}{|l|}{ (a) Univariate analysis } \\
\hline \multicolumn{2}{|l|}{ Beclin expression } \\
\hline$<20 \%(n=43)$ vs $\geqslant 20 \%(n=59)$ & $<0.0001$ \\
\hline \multicolumn{2}{|l|}{ Age } \\
\hline$<67$ years $(n=47)$ vs $\geqslant 67$ years $(n=55)$ & 0.867 \\
\hline \multicolumn{2}{|l|}{ Pathologic stage* } \\
\hline III and IV $(n=65)$ vs I and II $(n=36)$ & 0.008 \\
\hline
\end{tabular}

\begin{tabular}{|c|c|c|c|c|c|}
\hline \multirow[t]{2}{*}{ Prognosticator } & \multicolumn{5}{|c|}{ Multivariate } \\
\hline & Regression coefficient & Wald $\chi^{2}$ & $\mathrm{P}$ & Hazard ratio & $95 \%$ Confidential interval \\
\hline \multicolumn{6}{|c|}{ (b) Multivariate analysis } \\
\hline${ }^{*}$ Beclin $<20 \%$ & 2.33 & 21.45 & $<0.0001$ & 10.26 & $3.83-27.50$ \\
\hline Age $\geqslant 67$ years & -1.27 & 2.03 & 0.15 & 0.58 & $0.27-1.23$ \\
\hline Stage I and II & -0.57 & 6.24 & 0.013 & 0.25 & $0.09-0.75$ \\
\hline
\end{tabular}

The Cox proportional hazard model was applied to determine the prognostic significance of beclin-1 expression and of patient's age and pathologic stage at diagnosis.

${ }^{*}$ One case expressing $<20 \%$ beclin-1, for which was not known the pathologic stage, was excluded from the analysis.

overall survival probability was $58 \%$ (it was $44 \%$ at 5 year and $0 \%$ at 8 year). These correlations were statistically significant $(P<0.0001)$, based on both log-rank and Wilcoxon tests. To determine whether the prognostic value of beclin-1 persisted even when also patient's age and pathologic stage were considered, we performed a multivariate analysis using the Cox proportional hazards model. The Cox univariate analysis for these prognosticators confirmed a significant association with patient's survival of beclin-1 expression $(P<0.0001)$ and of pathologic stage $(P<0.008)$ (Table $4 a)$. We then carried out the Cox multiple regression to test the prognostic value of beclin-1 expression adjusting for patient's age and pathologic stage. Statistical data reported in Table $4 \mathrm{~b}$ indicate that the pathologic stage and the expression of beclin-1 are independent parameters significantly correlated with patients overall survival with a $P<0.01$ and $P<0.0001$, respectively. In particular, patients bearing a lymphoma-expressing beclin-1 (adjusted for the age and the stage) at low level ( $<20 \%$ positive cells) were associated with a shorter survival compared with those bearing a lymphoma highly expressing beclin-1 (hazard ratio of $10.3 ; 95 \%$ confidential interval ranging from 3.8 to 27.5 ).

\section{Beclin-1-Positivity Correlates with Favourable Prognosis when Associates with LC3-Positive Autophagy}

We interrogated regarding the functional significance of beclin-1 macro-aggregates in lymphoma cells. Depending on the partners involved in the protein macro-aggregate, beclin-1 can regulate autophagy, endosome formation or apoptosis. ${ }^{10,33,34}$ In this study, we considered the role of beclin-1 as an autophagy regulator, because this process has been shown to have a crucial role in lymphatic tissue homeostasis. ${ }^{6,7,27}$ On formation of the autophagosome, the microtubule-associated protein LC3 (isoform I) is modified to generate the truncated and lipidated isoform LC3-II, that is eventually inserted into the autophagosome membrane. ${ }^{14}$ Thus, autophagic vacuoles can be easily and reliably detected based on LC3 staining. ${ }^{35}$ To observe whether indeed beclin-1-positivity reflected ongoing autophagy, we monitored the presence of autophagic vacuoles visualized as LC3-positive puncta within tumour cells. We performed the LC3 immunostaining in 24 out of the 59 beclin-1-positive cases and in 12 out of the 43 beclin-1-negative cases, representative of the various histologic types and of the different pathologic stage and clinical outcome. Examples of LC3 staining in beclin-1-positive tumour cells are shown in Figure 3. The presence of LC3-positive discrete puncta reflects the presence of autophagic vacuoles, whereas a diffuse cytoplasmic staining is indicative of absence of autophagic vacuoles. ${ }^{35}$ We estimated the proportion of tumour cells showing LC3 as clearly detectable discrete spots in the cytoplasm. Data on beclin-1 and LC3 expression in relation to the clinical outcome and the histologic type of the cases examined are reported in Table 5a. We found that 17 out of 24 cases highly positive for beclin-1 were highly positive for LC3 also, assuming $\geqslant 20 \%$ of positive cells as the threshold. Of the 12 beclin-1negative cases examined, 7 showed $\geqslant 20 \%$ LC3positive cells. When data on LC3-positivity in beclin-1-positive non-Hodgkin lymphomas were correlated with the clinical outcome, it was found 

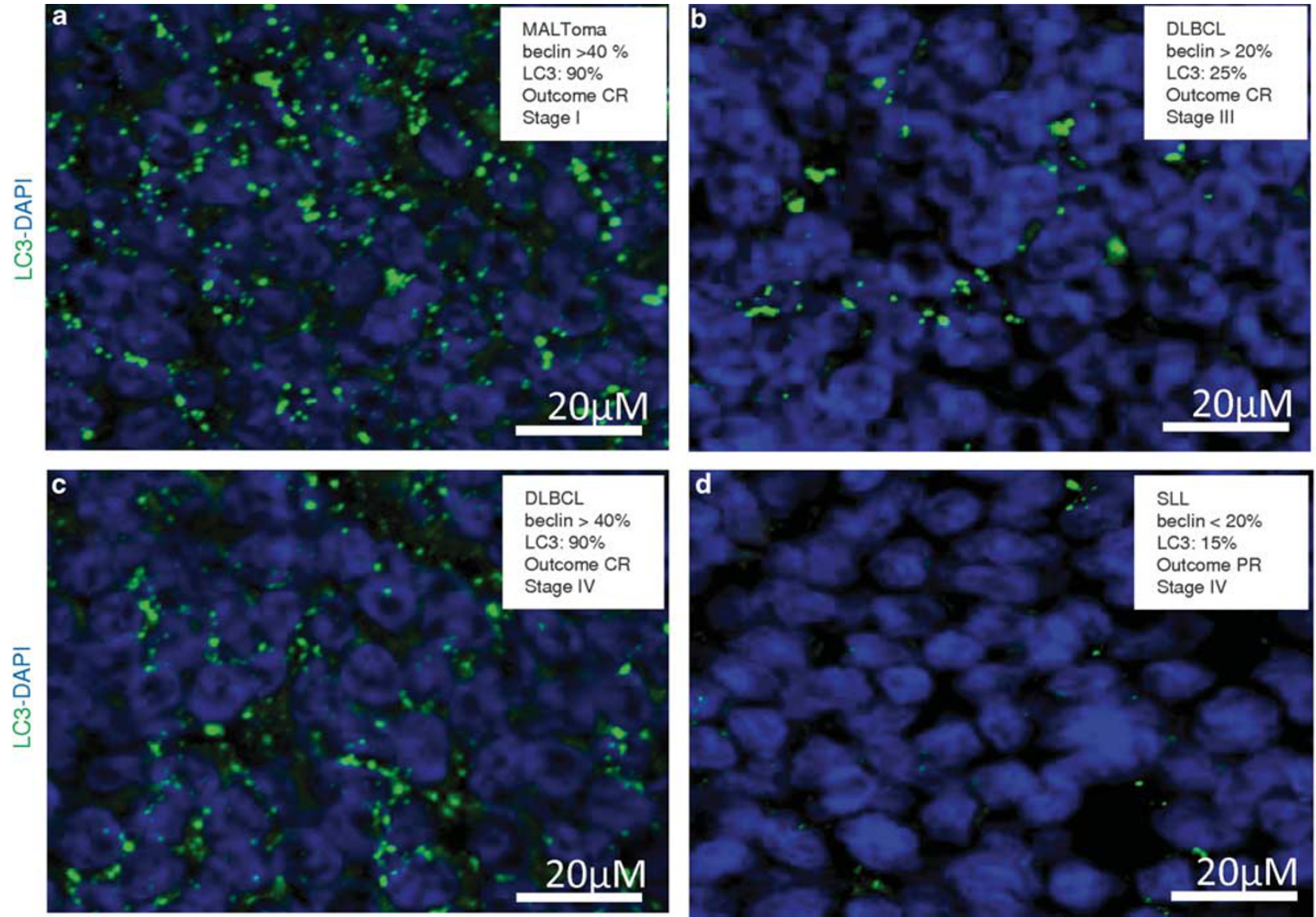

Figure 3 Immunofluorescence staining of LC3 in non-Hodgkin lymphoma tissue sections. Selection of representative cases. The histologic type (DLBCL, diffuse large B-cell lymphoma; MALToma, mucosa-associated lymphatic tissue lymphoma; SLL, small lymphocytic lymphoma), the clinical outcome (CR, complete remission; PR, partial remission), the pathologic stage and the positivity for beclin-1 aggregates and for vacuolar LC3 are reported. The nuclei are evidenced by DAPI staining. Examples of LC3-positive (panels a, b and c) and of LC3-negative (panel d) immunostaining.

that all the 5 exitus were associated with a very low $(<20 \%)$ proportion of LC3-positive tumour cells, whereas 17 out of $17(100 \%)$ of surviving patients (15 in complete remission and 2 in partial remission) were bearing a non-Hodgkin lymphoma with a high $(\geqslant 20 \%)$ proportion of LC3-positive tumour cells (Table 5b). This correlation was statistically significant $(P=0.0003)$. Statistics was then applied for all the 36 cases immunostained for LC3. It was found that of 24 LC3-positive non-Hodgkin lymphomas as many as $23(96 \%)$ were associated with survival at 8 year (Table 5c). Conversely, in the group of patients bearing an LC3-negative nonHodgkin lymphoma only $50 \%$ were alive at the end of the follow-up ( 2 complete remission and 4 partial remission out of 12). These correlations were statistically significant $(P=0.002)$.

\section{Expression and Immunolocalization of Beclin-1 and bcl-2 as Related to Vacuolar LC3 Staining}

Bcl-2 is one of the main interactor of beclin-1, and also exerts anti-apoptotic functions. ${ }^{36}$ The beclin-1/ bcl-2 system has a critical role in the complex regulation of both autophagy and apoptosis, two processes that determines the cells fate. ${ }^{34}$ In particular, when bcl-2 sequesters beclin-1, the autophagy interactome does not form. ${ }^{37}$ To learn more regarding the regulation of beclin-1-dependent autophagy in lymphoma cells, we performed a double immunostaining of bcl-2 and beclin-1 in selected cases of non-Hodgkin lymphoma showing concordance or discordance for both beclin-1 and LC3-positivity. Moreover, in a small subset of non-Hodgkin lymphomas for which the frozen biopsy was available, the expression of beclin-1 and of bcl-2 was assessed by western blotting. A representative selection of the examined cases is shown in Figure 4. The molecular forms revealed by the respective antibodies corresponded to the expected sizes for beclin-1 and bcl-2, which indirectly proved the specificity of the antibodies used in this study. In Figure 4, the positivity for beclin-1 aggregates, the extent of autophagy induction (based on percentage of vacuolar LC3-positive cells) and the clinical outcome are reported. In the majority of the cases, the relative intensity of the beclin-1 band well corresponded to the immunohistochemical assessment of its expression (as aggregates) in the tissue. Typically, vacuolar 
Table 5 Correlation of vacuolar LC3-positivity in a subgroup of non-Hodgkin lymphomas with clinical outcome in patients

\begin{tabular}{|c|c|c|c|c|c|c|c|}
\hline \multirow[t]{2}{*}{ Histologic type } & \multicolumn{2}{|c|}{ Beclin } & \multicolumn{2}{|c|}{$L C-3$} & \multirow[t]{2}{*}{ Survivors } & \multirow[t]{2}{*}{ Dead } & \multirow[t]{2}{*}{ No. of cases } \\
\hline & $<20 \%$ & $\geqslant 20 \%$ & $<20 \%$ & $\geqslant 20 \%$ & & & \\
\hline \multicolumn{8}{|c|}{ (a) Histologic type and positivity for beclin-1 and LC3 of the selected cases } \\
\hline DLBCL & 3 & 10 & 3 & 10 & 10 & 3 & 13 \\
\hline SLL & 5 & 5 & 5 & 5 & 8 & 2 & 10 \\
\hline MALToma & 1 & 3 & 0 & 4 & 4 & 0 & 4 \\
\hline FL IIIB & 0 & 4 & 1 & 3 & 3 & 1 & 4 \\
\hline MZL & 1 & 0 & 1 & 0 & 1 & 0 & 1 \\
\hline FL I and II & 2 & 1 & 1 & 2 & 3 & 0 & 3 \\
\hline PTCL & 0 & 1 & 1 & 0 & 0 & 1 & 1 \\
\hline Total & 12 & 24 & 12 & 24 & 29 & 7 & 36 \\
\hline
\end{tabular}

$\begin{array}{lllll}\% \text { LC3 positive } & \text { CR } & \text { PRitus } & \text { No. of cases }\end{array}$

(b) Subgroup of beclin-1-positive non-Hodgkin lymphomas analyzed for LC3 $\left(\chi^{2}=16.24\right.$; DF $\left.=2 ; P=0.0003\right)$

$\begin{array}{lrrrr}<20 \% & 1 & 1 & 5 & 7 \\ \geqslant 20 \% & 15 & 2 & 0 & 17 \\ \text { Total } & 16 & 3 & 5 & 24\end{array}$

(c) Subgroup of 24 beclin-1-positive plus 12 beclin-1-negative non-Hodgkin lymphomas analyzed for LC3 $\left(\chi^{2}=12.69 ; \mathrm{DF}=2 ; P=0.002\right)$

$\begin{array}{lrrrr}<20 \% & 2 & 4 & 6 & 12 \\ \geqslant 20 \% & 16 & 7 & 1 & 24 \\ \text { Total } & 18 & 11 & 7 & 36\end{array}$

Clinical outcome (CR, complete remission; PR, partial remission; exitus) was correlated with the proportion of cells showing vacuolar LC3.

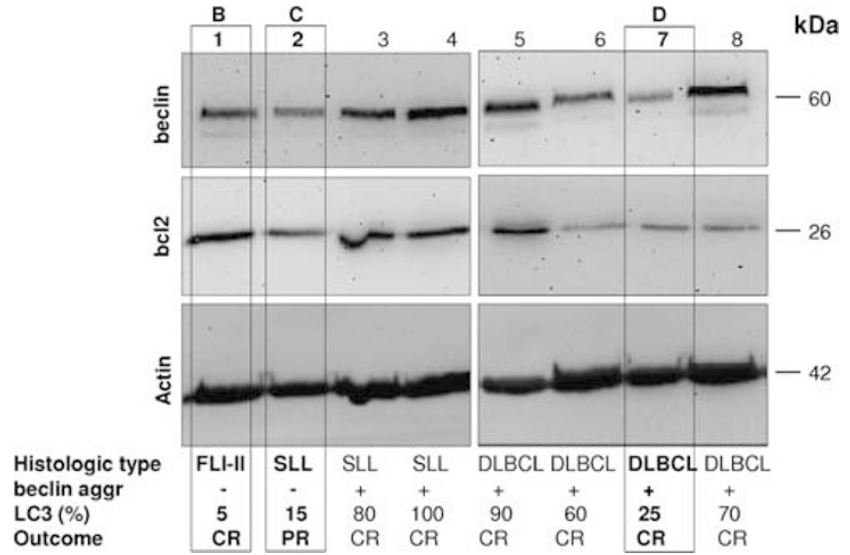

Figure 4 Western blotting analysis of the expression of beclin-1 and of bcl-2 proteins in non-Hodgkin lymphomas. Selection of representative cases. Tissue homogenates were subsequently probed for beclin-1, bcl-2 and actin (the latter was used as reference of homogenate protein loading). The molecular weight of proteins detected with the specific antibodies is indicated. The histologic type (DLBCL, diffuse large B-cell lymphoma; FL I and II, follicular lymphoma of grade I and II; SLL, small lymphocytic lymphoma), the clinical outcome (CR, complete remission; PR, partial remission), the positivity for beclin-1 aggregates and the percentage of cells positive for vacuolar LC3 are reported. (Immunofluorescence staining of bcl-2 and LC3 of tumour cases 1, 2 and 7 is shown in Figures 5b, c and d, respectively).

LC3-positivity and beclin-1 macro-aggregates were found in tumours expressing a high beclin-1/bcl-2 ratio (eg, lanes 4-6, 8 in Figure 4). Such typical situation is documented in the images of Figure 5a.
In this tumour, the proportion of cells containing beclin-1 aggregates is very high $(>40 \%)$. This tumour also expresses bcl-2 at a relatively high level in approximately $15 \%$ of the cells, and in a small percentage of tumour cells this protein colocalizes with beclin-1 (arrows). As a result, this tumour revealed to be positive for the autophagy marker LC3. We performed the immunostaining of beclin-1, bcl-2 and LC3 in three paradigmatic situations among the cases analyzed by western blotting (shown in Figure 4). The images in Figures 5b, c and $\mathrm{d}$, refer to the tumour cases 1,2 and 7 , respectively. (1) The tumour 'case 1' presented with a low percentage of cells expressing beclin-1 aggregates (15\%) and vacuolar LC3 (5\%). This tumour showed a very high expression of bcl-2, both by western blotting (Figure 4, lane 1) and by immunofluorescence (Figure 5b), which may account for the low level of autophagy activity (LC3 staining seems diffused in the cytoplasm). (2) By western blotting, the tumour 'case 2' expresses less beclin-1 and even much less bcl-2 as compared with case 1 (lane 2 vs lane 1 in Figure 4). By immunofluorescence, beclin-1 aggregates were undetectable and vacuolar LC3 was detected in 15\% of the cells. In the area where vacuolar LC3 was evident, bcl-2 was undetectable by immunofluorescence (not shown), whereas LC3 was undetectable in areas with cells in which bcl-2 was brightly evident (shown in Figure 5c). (3) By western blotting, the tumour 'case 7' expresses very low amount of both beclin-1 and bcl-2 proteins (Figure 4, lane 7). 

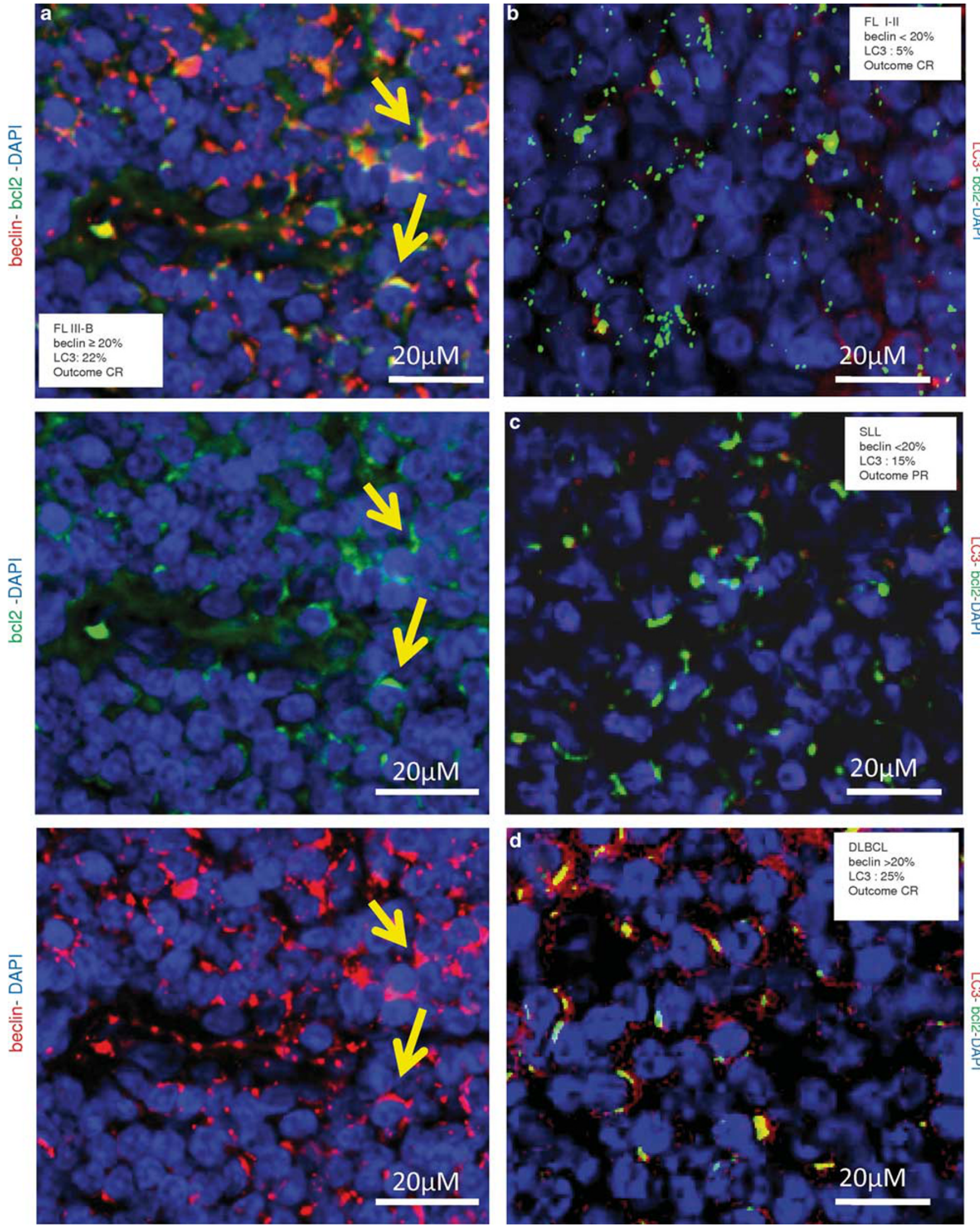

Figure 5 Immunofluorescence staining of beclin-1, bcl-2 and LC3 in non-Hodgkin lymphoma biopsies. Selection of representative cases. The histologic type (DLBCL, diffuse large B-cell lymphoma; FL, follicular lymphoma; SLL, small lymphocytic lymphoma), the clinical outcome (CR, complete remission; PR, partial remission), the pathologic stage, and the percentage of cells positive for beclin-1 aggregates and for vacuolar LC3 are reported. The nuclei are evidenced by DAPI staining. (a) Beclin-1 and bcl-2 staining (merge and single-channel as indicated). (b, c and d) LC3-bcl-2 co-staining of the tumour cases analyzed by western blotting in Figure 4, lanes 1, 2 and 7 , respectively. In (b) LC3 staining shows a cytoplasmic diffuse pattern. In (c) vacuolar LC3 staining is clearly evident in positive areas of the tumour. 
By immunofluorescence, this tumour was judged positive for beclin-1 aggregates and showed vacuolar LC3-positivity in $25 \%$ of the cells. Only a small proportion of cells showed positive for bcl-2. Thus, the tissue expression of bcl-2 and of vacuolar LC3 seems mutually exclusive. The above data also outline the warning that western blotting determination of beclin-1 may not be sufficient to draw a definitive conclusion regarding the level of autophagy. In fact, whatever the level of beclin-1 and bcl-2 proteins expressed by the tumour, for the assessment of autophagy it seems mandatory to study their relative localization within the cell, along with a reliable autophagy marker such as LC3.

\section{Discussion}

In this work, the tissue expression of beclin-1 was analyzed in a large series (102 cases) of non-Hodgkin lymphomas and correlated with clinical outcome in patients. By immunofluorescence, beclin-1 protein was either distributed diffusely in the cytoplasm and, therefore, faintly/not all detectable, or confined to the perinuclear region as large and brilliant puncta suggestive of macro-aggregate reactivity. The latter immunofluorescence pattern likely reflected the engagement of beclin-1 in macro-complexes associated with autophagy activity, ${ }^{23}$ as also testified by the concomitant presence of LC3positive structures. In 59 non-Hodgkin lymphomas, beclin-1 macro-aggregates were observed in a percentage of cells ranging from 20 to $90 \%$. It is to be stressed that in our study only tumour cells were considered for beclin-1 expression. Reactive macrophages or T-lymphocytes infiltrating the tumour area were identified and excluded from the count of beclin-1-positive cells. The expression of beclin-1 was not correlated with patient's individual characteristics such as gender and age at the time of diagnosis, nor was it with a particular histologic type. Setting the cut-off at $20 \%$ of positive cells (in terms of beclin-1 macro-aggregates), no statistically significant correlation was found between beclin-1 expression and the pathologic stage at diagnosis. It is noteworthy that non-Hodgkin lymphomas in which $\geqslant 20 \%$ of tumour cells expressed high level of beclin-1 macro-aggregates were associated with patient survival (33 complete remission and 21 partial remission out of 59). More strikingly, of the 39 patients who underwent complete remission as many as 33 were bearing a beclin-1-positive nonHodgkin lymphoma and only 6 were bearing a beclin-1-negative non-Hodgkin lymphoma. Moreover, 24 out of 43 patients bearing a non-Hodgkin lymphoma with $<20 \%$ beclin-1-positive cells died during the course of the study. These correlations were statistically highly significant $(P<0.0001)$. Consistently, patients bearing a non-Hodgkin lymphoma with $\geqslant 20 \%$ cells expressing active beclin-1 showed greater than twofolds overall survival probability toward patients bearing a non-Hodgkin lymphoma in which $<20 \%$ of tumour cells were positive for this autophagy protein $(P<0.0001)$, regardless of the histologic type (whether aggressive or indolent), the clinical assessment (pathological staging) at diagnosis and of the therapy regimen. Pathologic stage was also significantly correlated with overall survival, as expected. Cox univariate analyses confirmed the prognostic value of beclin-1 expression $(P<0.0001)$ and of pathologic stage $(P<0.008)$. The Cox multivariate analysis for the variables patient's age, pathologic stage and tumour cell beclin-1 expression revealed that both the pathologic stage and beclin-1 expression were independent prognosticators of overall survival with $P<0.002$ and $P<0.0001$, respectively. Low expression of beclin-1 in the tumour context was significantly associated with shorter patient's survival (hazard ratio of 10.3). On the basis of the Wald $\chi^{2}$ value, it seems that the level of beclin-1 expression is the most important variable (among the three considered) influencing patient's survival.

We note that five patients bearing a non-Hodgkin lymphomas with $\geqslant 20 \%$ of beclin-1-positive cells deceased within the follow-up period. This fact was not related to a particular histologic type, regardless of whether it belonged to the indolent (low-malignancy) or aggressive (highly malignancy) subgroup (one FL, one SLL, one PTCL and two DLBCL), and was not associated with the patient's age or the pathologic stage at diagnosis or with a specific therapy regimen (regardless of whether this included or not rituximab). Beside the possibility that exitus occurred because of patient's clinical conditions, we analyzed the molecular basis that could explain why in some tumours the expression of beclin-1 was not 'per se' sufficient to warrant the beneficial effects of chemotherapy. Assuming that beclin-1 main function was to drive autophagy, and that autophagy was having a positive role in the response to chemotherapy treatments, we considered the possibility that failure in chemotherapy response could arise from impaired (or insufficient) induction of autophagy in tumour cells in spite of the high expression of beclin-1 macro-aggregates. We therefore searched for signs of ongoing autophagy in a number of beclin-1-positive and beclin-1negative non-Hodgkin lymphomas, representative of the different clinical outcome. The vacuolar pattern of LC3 immunolocalization was assumed to prove ongoing autophagy. ${ }^{34}$ It is noteworthy that the five beclin-1-positive non-Hodgkin lymphomas associated with the patients who died during the study resulted negative for vacuolar LC3 staining. Thus, in these tumours autophagy was defective in spite of the presence of beclin-1 macro-aggregates. However, a high concordance between beclin-1-positivity and LC3-positivity was observed in the large majority of the cases. Moreover, the presence of LC3-positive vacuoles in relation to the expression and localization of beclin-1 and bcl-2 was compa- 
tible with the predicted model of bcl-2-mediated regulation of beclin-1-dependent autophagy. ${ }^{37}$ Our data are in agreement with the observation of Saeki et $a l,{ }^{38}$ who reported an increase of basal autophagy in leukaemic cells after downregulation of bcl-2 expression. In a few cases, tumours with a low percentage of beclin-1-positive cells presented with a relative high proportion of LC-3-positive cells, which is suggestive of the activation of a beclin-1independent autophagy pathway. ${ }^{39}$ Although the number of cases examined for LC3-positivity was relatively small (36 cases), and despite they were arbitrarily chosen as representative of the whole series, the data obtained indicate (1) that beclin-1 macro-aggregates associate with functional autophagy (as assessed by vacuolar LC3-positivity) in the majority of the cases (71\%), and (2) that upregulation of autophagy (assumed as $\geqslant 20 \%$ LC3-positive cells) in the tumour context associates with a favourable outcome.

According to the proposed model of autophagy modulation during the various steps of carcinogenesis and tumour progression, ${ }^{19}$ we give the following interpretation of our data. In the early step of lymphomagenesis, defective expression of beclin-1 and/or hyper-expression of bcl-2 inhibit(s) autophagy (and apoptosis) and favour(s) tumour development. Thereafter, bcl-2 downregulation and/or beclin-1 upregulation concur(s) to the hyper-induction of autophagy, which confers an advantage to the tumour cells enabling them to survive in spite of the high cellular density and the low supply of nutrients. These cells are, however, unable to cope with an additional stress such as that provoked by the chemotherapeutic drug. Cells in which autophagy is basally upregulated may succumb in response to drugs that either over stimulate or, by contrary, totally inhibit autophagy. The following observations give support to our hypothesis: (1) rapamycin is a natural inhibitor of the kinase mTOR (mammalian target of rapamycin), which normally represses autophagy. In a phase II clinical trial, the rapamycin derivative temsirolimus revealed significant clinical activity in patients with relapsed mantle cell lymphoma. ${ }^{40}$ This drug was shown to hyper-induce autophagy and to elicit synergistic anti-tumour effect with vorinostat at low doses in mantle cell lymphoma cells; ${ }^{41}$ (2) chloroquine is a well-known lysosomotropic anti-malarial drug that impairs intralysosomal proteolysis by alkalinizing the vacuolar $\mathrm{pH}$. In myc-overexpressing transgenic mice that model human Burkitt's lymphoma, chloroquine administration prevented the development of myc-induced lymphomas by killing precancerous B lymphocytes through a p53-dependent and caspase-independent death pathway. ${ }^{42}$ Chloroquineinduced autophagy, yet chronic administration eventually impaired the degradation of autophagocytosed material and resulted in cell death of premalignant myc-overexpressing B cells. ${ }^{42}$ Thus, autophagy elicits a pro-survival function as long as the lysosome is able to clear the incoming autophagocytosed material, whereas it switches to a prodeath function if the digestive properties of lysosomes are compromised. Yet another investigation has shown that inhibition of autophagy by chloroquine enhances the pro-apoptotic effects of the histone deacetylase suberoylanide hydroxamic acid in primary chronic myelogenous leukaemia cells from patients clinically refractory to imatinib. ${ }^{43}$ Interestingly, the pro-death synergism between these two drugs was associated with disruption of lysosomal membrane integrity and cathepsin D-mediated degradation of thioredoxin, a key regulator of cellular redox status. ${ }^{43}$ These findings underscore the complexity of the role(s) of autophagy in anticancer drug response, which likely depend on the actual level of autophagy in the cell and the cytotoxic mechanism of the therapeutic drug.

In conclusion, our data clearly show for the first time a significant correlation of beclin-1 aggregates with autophagy in non-Hodgkin lymphoma cells, and with a favourable clinical outcome in chemotherapy-treated patients. The present findings outline the relevance of autophagy proteins as potential targets for the treatment of this heterogeneous group of cancer. Therefore, the introduction of autophagyenhancer drugs in the chemotherapy cocktail should improve the chance to cure non-Hodgkin lymphoma. Beclin-1-independent pathways of autophagy are being elucidated. ${ }^{39}$ We suggest that in nonHodgkin lymphomas not expressing beclin-1 the chemotherapy regimen should include drugs able to induce beclin-1-independent autophagy. Finally, the data here reported show that the immunohistochemical detection of beclin-1 (and of LC3) in nonHodgkin lymphoma has a great prognostic potential. In the future, we plan to extend our analysis to a larger cohort of patients, including other types of lymphoma such as mantle cell lymphoma and Hodgkin lymphoma.

\section{Acknowledgements}

This work was supported by grants from Regione Piemonte (Ricerca Sanitaria Finalizzata), Fondazione Cassa di Risparmio di Torino (Torino, Italy) and Lega Italiana Lotta contro i Tumouri (Novara, Italy). CP was supported with a fellowship from Associazione per la Ricerca Medica HyppocratesRhazi (Novara, Italy). The authors are grateful to Drs E Bartoli, G Gaidano, G Avanzi and A Bertoncelli for their kind permission to analyze the clinical files of the patients. The microscopy bio-imaging facility is sponsored by Comoli-Ferrari \& C. SpA (Novara, Italy).

\section{Disclosure/conflict of interest}

The authors declare no conflict of interest. 


\section{References}

1 Gao X, Zacharek A, Salkowski A, et al. Los of heterozygosity of the BRCA1 and other loci on chromosome $17 \mathrm{q}$ in human prostate cancer. Cancer Res 1995;55:1002-1005.

2 Futreal PA, Söderkvist P, Marks JR, et al. Detection of frequent allelic loss on proximal chromosome $17 q$ in sporadic breast carcinoma using microsatellite length polymorphisms. Cancer Res 1992;52:2624-2627.

3 Saito H, Inazawa J, Saito S, et al. Detailed deletion mapping of chromosome $17 \mathrm{q}$ in ovarian and breast cancers: 2-cM region on 17q21.3 often and commonly deleted in tumors. Cancer Res 1993;53:3382-3385.

4 Russell SE, Hickey GI, Lowry WS, et al. Allele loss from chromosome 17 in ovarian cancer. Oncogene 1990;5:1581-1583.

5 Eccles DM, Russell SE, Haites NE, et al. Early loss of heterozygosity on $17 q$ in ovarian cancer. The Abe Ovarian Cancer Genetics Group. Oncogene 1992;7:2069-2072.

6 Yue Z, Jin S, Yang C, et al. Beclin 1, an autophagy gene essential for early embryonic development, is a haploinsufficient tumor suppressor. Proc Natl Acad Sci USA 2003;100:15077-15082.

$7 \mathrm{Qu} \mathrm{X,} \mathrm{Yu} \mathrm{J,} \mathrm{Bhagat} \mathrm{G,} \mathrm{et} \mathrm{al.} \mathrm{Promotion} \mathrm{of} \mathrm{tumorigenesis}$ by heterozygous disruption of the beclin 1 autophagy gene. J Clin Invest 2003;112:1809-1820.

8 Liang XH, Kleeman LK, Jiang HH, et al. Protection against fatal Sindbis virus encephalitis by beclin, a novel Bcl-2interacting protein. J Virol 1998;72:8586-8596.

9 Itakura $\mathrm{E}$, Kishi $\mathrm{C}$, Inoue $\mathrm{K}$, et al. Beclin 1 forms two distinct phosphatidylinositol 3-kinase complexes with mammalian Atg14 and UVRAG. Mol Biol Cell 2008;19:5360-5372.

10 Zhong Y, Wang QJ, Li X, et al. Distinct regulation of autophagic activity by Atg14L and Rubicon associated with beclin1-phosphatidylinositol-3-kinase complex. Nat Cell Biol 2009;11:468-476.

11 Klionsky DJ. Autophagy: from phenomenology to molecular understanding in less than a decade. Nat Rev Mol Cell Biol 2007;8:931-937.

12 Kihara A, Kabeya Y, Ohsumi Y, et al. Beclin-phosphatidylinositol 3-kinase complex functions at the transGolgi network. EMBO Rep 2001;2:330-335.

13 Kabeya Y, Mizushima N, Ueno T, et al. LC3, a mammalian homologue of yeast Apg8p, is localized in autophagosome membranes after processing. EMBO J 2000;19:5720-5728.

14 Noda T, Fujita N, Yoshimori T. The late stages of autophagy: how does the end begin? Cell Death Differ 2009;16:984-990.

15 Liao XH, Majithia A, Huang X, et al. Growth control via TOR kinase signaling, an intracellular sensor of amino acid and energy availability, withcrosstalk potential to proline metabolism. Amino Acids 2008;35:761-770.

16 Levine B, Kroemer G. Autophagy in the pathogenesis of disease. Cell 2008;132:27-42.

17 Eisenberg-Lerner A, Bialik S, Simon HU, et al. Life and death partners: apoptosis, autophagy and the cross-talk between them. Cell Death Differ 2009; 16:966-975.

18 He C, Levine B. The beclin 1 interactome. Curr Opin Cell Biol 2010;22:140-149.

19 Morselli E, Galluzzi L, Kepp O, et al. Anti- and protumor functions of autophagy. Biochim Biophys Acta 2009;1793:1524-1532.
20 Apel A, Zentgraf H, Büchler MW, et al. Autophagy-A double-edged sword in oncology. Int $J$ Cancer 2009;125:991-995.

21 Kondo Y, Kanzawa T, Sawaya R, et al. The role of autophagy in cancer development and response to therapy. Nat Rev Cancer 2005;5:726-734.

22 Amaravadi RK, Yu D, Lum JJ, et al. Autophagy inhibition enhances therapy-induced apoptosis in a Myc-induced model of lymphoma. J Clin Invest 2007;117:326-336.

23 Trincheri NF, Follo C, Nicotra G, et al. Resveratrolinduced apoptosis depends on the lipid kinase activity of Vps34 and on the formation of autophagolysosomes. Carcinogenesis 2008;29:381-389.

24 Shingu T, Fujiwara K, Bögler O, et al. Inhibition of autophagy at a late stage enhances imatinib-induced cytotoxicity in human malignant glioma cells. Int J Cancer 2009;124:1060-1071.

$25 \mathrm{Li} \mathrm{C}$, Capan E, Zhao Y, et al. Autophagy is induced in CD4+ $\mathrm{T}$ cells and important for the growth factor-withdrawal cell death. J Immunol 2006;177: 5163-5168.

26 Pua HH, Dzhagalov I, Chuck M, et al. A critical role for the autophagy gene Atg5 in $\mathrm{T}$ cell survival and proliferation. J Exp Med 2007;204:25-31.

27 Arsov I, Li X, Matthews G, et al. BAC-mediated transgenic expression of fluorescent autophagic protein beclin 1 reveals a role for beclin 1 in lymphocyte development. Cell Death Differ 2008;15:1385-1395.

28 Harris NL, Jaffe ES, Diebold J, et al. World Health Organization classification of neoplastic diseases of the hematopoietic and lymphoid tissues: report of the Clinical Advisory Committee meeting-Airlie House, Virginia, November 1997. J Clin Oncol 1999;17:3835-3849.

29 Castino R, Peracchio C, Salini A, et al. Chemotherapy drug response in ovarian cancer cells strictly depends on a cathepsin D-Bax activation loop. J Cell Mol Med 2009;13:1096-1109.

30 Kaplan EL, Meier P. Nonparametric estimation from incomplete observations. J Am Stat Assoc 1958;53: 457-481.

31 Pileri SA, Ascani S, Sabattini E, et al. The pathologist's view point. Part I indolent lymphomas. Haematologica 2000a;85:1291-1307.

32 Pileri SA, Ascani S, Sabattini E, et al. The pathologist's view point. Part II -aggressive lymphomas. Haematologica 2000b;85:1308-1321.

33 Itakura E, Mizushima N. Atg14 and UVRAG: mutually exclusive subunits of mammalian Beclin 1-PI3K complexes. Autophagy 2009;5:534-536.

34 Proikas-Cezanne T, Pfisterer SG. Assessing mammalian autophagy by WIPI-1/Atg18 puncta formation. Methods Enzymol 2009;452:247-260.

35 Adams JM, Cory S. The Bcl-2 apoptotic switch in cancer development and therapy. Oncogene 2007;26: 1324-1337.

36 Levine B, Sinha S, Kroemer G. Bcl-2 family members: dual regulators of apoptosis and autophagy. Autophagy 2008;4:600-606.

37 Pattingre S, Tassa A, Qu X, et al. Bcl-2 antiapoptotic proteins inhibit beclin 1-dependent autophagy. Cell 2005;122:927-939.

38 Saeki K, Yuo A, Okuma E, et al. Bcl-2 down-regulation causes autophagy in a caspase-independent manner in human leukemic HL60 cells. Cell Death Differ 2000;7:1263-1269. 
39 Scarlatti F, Maffei R, Beau I, et al. Role of noncanonical beclin 1-independent autophagy in cell death induced by resveratrol in human breast cancer cells. Cell Death Differ 2008;15:1318-1329.

40 Witzig TE, Geyer SM, Ghobrial I, et al. Phase II trial of single-agent temsirolimus (CCI-779) for relapsed mantle cell lymphoma. J Clin Oncol 2005; 23:5347-5356.

41 Yazbeck VY, Buglio D, Georgakis GV, et al. Temsirolimus downregulates p21 without altering cyclin D1 expression and induces autophagy and synergizes with vorinostat in mantle cell lymphoma. Exp Hematol 2008;36:443-450.

42 Maclean KH, Dorsey FC, Cleveland JL, et al. Targeting lysosomal degradation induces p53-dependent cell death and prevents cancer in mouse models of lymphomagenesis. J Clin Invest 2008;118:79-88.

43 Carew JS, Nawrocki ST, Kahue CN, et al. Targeting autophagy augments the anticancer activity of the histone deacetylase inhibitor SAHA to overcome Bcr-Abl-mediated drug resistance. Blood 2007;110: 313-322. 